\title{
sciendo
}

Int. J. of Applied Mechanics and Engineering, 2018, vol.23, No.4, pp.873-884

DOI: $10.2478 /$ ijame-2018-0048

\section{A STUDY ON VIBRATION OF TAPERED NON-HOMOGENEOUS RECTANGULAR PLATE WITH STRUCTURAL PARAMETERS}

\author{
N. KAUR* \\ Department of Mathematics, Statistics and Physics \\ Punjab Agricultural University \\ Ludhiana, Punjab, INDIA \\ E-mail: narinder89.kaur@gmail.com \\ A. SINGHAL \\ Department of Mathematics \\ Dr. Akhilesh Das Gupta Institute of Technology and Management \\ New Delhi, INDIA \\ E-mail: ashishsinghal11@gmail.com \\ A. KHANNA \\ Department of Mathematics, D.A.V. College \\ Sadhaura, Yamunanagar, Haryana, INDIA \\ E-mail: rajieanupam@gmail.com
}

\begin{abstract}
Effects of structural parameters on the vibration of a tapered non-homogeneous rectangular plate with different combinations of boundary conditions are discussed. Tapering in the plate is assumed to be sinusoidal in the $x$-direction. Here, temperature variation and non-homogeneity in the plate material are also considered sinusoidal in the $x$-direction. The Rayleigh-Ritz method is used to calculate the frequency parameter for the first two modes of vibration for different values of the structural parameters, i.e. the taper parameter, thermal gradient, aspect ratio and non-homogeneity constant. Results are obtained for three boundary conditions, i.e. clamped boundary (C-C-C-C), simply supported boundary (SS-SS-SS-SS) and clamped-simply supported boundary (CSS-C-SS). Numerical values of the frequency parameter are given in a compact tabular form.
\end{abstract}

Key words: vibration, non-homogeneity, thermal gradient, taper parameter, clamped, simply supported, aspect ratio.

\section{Introduction}

In the construction of structures, machines and development of other mechanical designs, engineers and researchers always need to know the vibration characteristics of the system. The importance of studying vibrations of non-homogeneous tapered plates which are widely used in the construction of ships, aircrafts, bridges, etc., cannot be neglected. Non-homogeneity along with tapering in the plates makes the material lighter and stronger. The main purpose of the study on vibrations is to reduce unnecessary and uncontrolled vibration by making proper and accurate designs of machines and structures. In the modern era of technology, most of the structures and equipment work in high temperature fields with various boundary conditions. Therefore it becomes necessary to study the impact of the temperature variation on the vibrational properties of the structures.

\footnotetext{
* To whom correspondence should be addressed
} 
Since half of the $19^{\text {th }}$ century, a lot of work has been done in the field of vibration of plates. One dimensional thickness variations were considered by many researchers. But nowadays many researchers are working on two dimensional thickness variations of the plates with different boundary conditions. Boundary conditions are essential to determine the mathematical solutions to many physical problems.

In Leissa [1] published a collection of research papers in his monograph, in which thermal effects on different shapes of the plate with various boundary conditions were discussed. In this work, the author discussed various combinations of clamped, simply-supported and free edge boundary conditions. Sobotka [2] discussed the free vibrations of orthotropic visco-elastic plates with various combinations of boundary conditions. Tomar and Gupta [3] investigated the frequencies of an orthotropic plate of varying thickness under thermal conditions. In Tomar et al. [4] again discussed the free vibrations of an infinite plate with parabolically varying thickness. Cheung and Zhou [5] studied the free vibrations of a tapered rectangular plate using the Rayleigh-Ritz method with a set of beam functions. In Lal [6] studied the transverse vibrations of an orthotropic non-uniform rectangular plate with continuously varying density. Li [7] analyzed the vibrations of a rectangular plate with general elastic boundary support. Gupta et al. [8] analyzed the transverse vibrations of a non-homogeneous orthotropic visco-elastic circular plate of varying parabolic thickness. Gupta and Sharma [9] studied the thermal effect on the frequencies of a trapezoidal plate with varying thickness and density of the material. In Khanna and Singhal [10] studied the vibrations of a viscoelastic plate with bi-dimensional thickness and temperature variations.

Here, the authors investigated the effect of various structural parameters on vibrations of a nonhomogeneous tapered isotropic rectangular plate. Tapering in thickness is considered sinusoidal. Also, the temperature variation and non-homogeneity are considered sinusoidal in the $x$-direction. A tabular presentation of numerical values of the first two modes of the frequency parameter at various values of the structural parameters for three boundary conditions is given.

\section{Analysis of motion and assumptions required}

The fourth order differential equation of motion for an isotropic rectangular plate in the Cartesian system is [15]

$$
\begin{aligned}
& \widetilde{D}\left[D_{1}\left(\frac{\partial^{4} w}{\partial x^{4}}+2 \frac{\partial^{4} w}{\partial x^{2} \partial y^{2}}+\frac{\partial^{4} w}{\partial y^{4}}\right)+2 \frac{\partial D_{1}}{\partial x}\left(\frac{\partial^{3} w}{\partial x^{3}}+2 \frac{\partial^{3} w}{\partial x \partial y^{2}}\right)+2 \frac{\partial D_{1}}{\partial y}\left(\frac{\partial^{3} w}{\partial y^{3}}+2 \frac{\partial^{3} w}{\partial y \partial x^{2}}\right)+\right. \\
& \left.+\frac{\partial^{2} D_{1}}{\partial x^{2}}\left(\frac{\partial^{2} w}{\partial x^{2}}+v \frac{\partial^{2} w}{\partial y^{2}}\right)+\frac{\partial^{2} D_{1}}{\partial y^{2}}\left(\frac{\partial^{2} w}{\partial y^{2}}+v \frac{\partial^{2} w}{\partial x^{2}}\right)+2(1-v) \frac{\partial^{2} D_{1}}{\partial x \partial y} \frac{\partial^{2} w}{\partial x \partial y}\right]+\rho h \frac{\partial^{2} w}{\partial t^{2}}=0
\end{aligned}
$$

where $D_{1}=\frac{E h^{3}}{12\left(1-v^{2}\right)}$ is the flexural rigidity of the plate material, $\widetilde{D}$ is the visco-elastic operator, $w(x, y, t)$ is the deflection, $v$ is the Poisson ratio, $\rho$ is the density of the plate material, $\mathrm{E}$ is the Young's modulus and $h$ is the thickness of the plate.

The deflection can be considered as a product of two functions as [14]

$$
w(x, y, t)=W(x, y) \times T(t)
$$

where $W(x, y)$ is the deflection function of the rectangular plate and $T(t)$ is a time function.

On using Eq.(2.2) in Eq.(2.1), we obtain 


$$
\begin{aligned}
& {\left[D_{1}\left(\frac{\partial^{4} W}{\partial x^{4}}+2 \frac{\partial^{4} W}{\partial x^{2} \partial y^{2}}+\frac{\partial^{4} W}{\partial y^{4}}\right)+2 \frac{\partial D_{1}}{\partial x}\left(\frac{\partial^{3} W}{\partial x^{3}}+2 \frac{\partial^{3} W}{\partial x \partial y^{2}}\right)+2 \frac{\partial D_{1}}{\partial y}\left(\frac{\partial^{3} W}{\partial y^{3}}+2 \frac{\partial^{3} W}{\partial y \partial x^{2}}\right)+\right.} \\
& \left.+\frac{\partial^{2} D_{1}}{\partial x^{2}}\left(\frac{\partial^{2} W}{\partial x^{2}}+v \frac{\partial^{2} W}{\partial y^{2}}\right)+\frac{\partial^{2} D_{1}}{\partial y^{2}}\left(\frac{\partial^{2} W}{\partial y^{2}}+v \frac{\partial^{2} W}{\partial x^{2}}\right)+2(1-v) \frac{\partial^{2} D_{1}}{\partial x \partial y} \frac{\partial^{2} W}{\partial x \partial y}\right] / \rho h W=-\left(\frac{\partial^{2} T / \partial t^{2}}{\widetilde{D} T}\right),
\end{aligned}
$$

Eq.(2.3) is satisfied if both of its sides are equal to a constant $p^{2}$, i.e.

$$
\begin{aligned}
& {\left[D_{l}\left(\frac{\partial^{4} W}{\partial x^{4}}+2 \frac{\partial^{4} W}{\partial x^{2} \partial y^{2}}+\frac{\partial^{4} W}{\partial y^{4}}\right)+2 \frac{\partial D_{l}}{\partial x}\left(\frac{\partial^{3} W}{\partial x^{3}}+2 \frac{\partial^{3} W}{\partial x \partial y^{2}}\right)+2 \frac{\partial D_{l}}{\partial y}\left(\frac{\partial^{3} W}{\partial y^{3}}+2 \frac{\partial^{3} W}{\partial y \partial x^{2}}\right)+\right.} \\
& \left.+\frac{\partial^{2} D_{l}}{\partial x^{2}}\left(\frac{\partial^{2} W}{\partial x^{2}}+v \frac{\partial^{2} W}{\partial y^{2}}\right)+\frac{\partial^{2} D_{l}}{\partial y^{2}}\left(\frac{\partial^{2} W}{\partial y^{2}}+v \frac{\partial^{2} W}{\partial x^{2}}\right)+2(1-v) \frac{\partial^{2} D_{l}}{\partial x \partial y} \frac{\partial^{2} W}{\partial x \partial y}\right]-\rho p^{2} h W=0
\end{aligned}
$$

and $\quad \frac{\partial^{2} T}{\partial t^{2}}+p^{2} \widetilde{D} T=0$

Equation (2.4) is a differential equation of motion and Eq.(2.5) is a differential equation of time function for a rectangular plate.

\subsection{Assumption for thickness variation}

In this paper, the authors assumed a new variation in thickness of the rectangular plate. It is assumed that the thickness of the rectangular plate varies sinusoidally in the $x$-direction, i.e.

$$
h=h_{0}\left(1+\beta \sin \left(\pi \frac{x}{a}\right)\right)
$$

where $a$ is the length of the rectangular plate, $\beta(0 \leq \beta \leq 1)$ is the taper parameter in the $x$-direction and $h_{0}$ is the thickness of the plate at $x=0$ and $x=a$. It is evident from the expression of the thickness in Eq.(2.6) that the minimum value of the thickness is $h_{0}$ (at $x=0$ and $a$ ) while the maximum value of the thickness is $2 h_{0}$ (at $x=a / 2$ and $\beta=1)$.

\subsection{Assumption for temperature variation}

Since temperature affects the vibrational properties of the rectangular plate, different combinations of temperature variation have been discussed by various authors in the available literature. In addition, the authors assumed sinusoidal temperature variation which has never been discussed earlier

$$
\tau=\tau_{0}\left(1-\sin \left(\pi \frac{x}{2 a}\right)\right)
$$

where $\tau$ denotes the temperature excess above the reference temperature at any point on the plate and $\tau_{0}$ denotes the temperature excess above the reference temperature at $x=0$.

The temperature dependence of the modulus of elasticity can be expressed as follows [12] 


$$
\mathrm{E}=E_{0}(1-\gamma \tau)
$$

where $E_{0}$ is the value of the Young's modulus at reference temperature and $\gamma$ is the slope of the variation of $\mathrm{E}$ and $\tau$. On using Eq.(2.7) in Eq.(2.8), one obtains

$$
\mathrm{E}=E_{0}\left[1-\alpha\left(1-\sin \left(\pi \frac{x}{2 a}\right)\right)\right]
$$

where, $\alpha=\gamma \tau_{0}(0 \leq \alpha \leq 0.8)$ is the thermal gradient.

From the values of $h$ and $\mathrm{E}$, the expression of the flexural rigidity $\left(D_{l}\right)$ becomes

$$
D_{1}=\frac{E_{0}\left[1-\alpha\left(1-\sin \left(\pi \frac{x}{2 a}\right)\right)\right] h_{0}^{3}\left(1+\beta \sin \left(\pi \frac{x}{a}\right)\right)^{3}}{12\left(1-v^{2}\right)} .
$$

\subsection{Assumption for non-homogeneity in the plate material}

Non-homogeneity in the plate material is characterized by density. The authors assumed that density varies sinusoidally in the $x$-direction, i.e.

$$
\rho=\rho_{0}\left(1+\alpha_{1} \sin \left(\pi \frac{x}{a}\right)\right)
$$

where $\rho$ is the density of the plate material, $\rho_{0}$ is the density at $x=0$ and $\alpha_{l}$ is the non-homogeneity constant $\left(0 \leq \alpha_{1} \leq 1.0\right)$.

\subsection{Boundary conditions}

In this study, the authors discussed the vibration of a rectangular plate for three different boundary conditions which are usually seen in most of the mechanical designs and structures, i.e. C-C-C-C, SS-SS-SS-SS and C-SS-C-SS.

In order to discuss all these BCs, the following expressions for the deflection function are taken [16]:

For C-C-C-C, $\quad W=\left[\left(\frac{x}{a}\right)\left(\frac{y}{b}\right)\left(1-\frac{x}{a}\right)\left(1-\frac{y}{b}\right)\right]^{2} \times\left[A_{1}+A_{2}\left(\frac{x}{a}\right)\left(\frac{y}{b}\right)\left(1-\frac{x}{a}\right)\left(1-\frac{y}{b}\right)\right]$.

For SS-SS-SS-SS, $W=\left[\left(\frac{x}{a}\right)\left(\frac{y}{b}\right)\left(1-\frac{x}{a}\right)\left(1-\frac{y}{b}\right)\right] \times\left[A_{1}+A_{2}\left(\frac{x}{a}\right)\left(\frac{y}{b}\right)\left(1-\frac{x}{a}\right)\left(1-\frac{y}{b}\right)\right]$.

For C-SS-C-SS, $\quad W=\left[\left(\frac{x}{a}\right)^{2}\left(\frac{y}{b}\right)\left(1-\frac{x}{a}\right)^{2}\left(1-\frac{y}{b}\right)\right] \times\left[A_{1}+A_{2}\left(\frac{x}{a}\right)\left(\frac{y}{b}\right)\left(1-\frac{x}{a}\right)\left(1-\frac{y}{b}\right)\right]$.

\section{Solution of frequency parameter}

The Rayleigh Ritz technique is applied to solve the frequency equation. In this method, the maximum 
strain energy $\left(S_{E}\right)$ must be equal to the maximum kinetic energy $\left(K_{E}\right)$. So it is necessary for the problem under consideration that [11]

$$
\delta\left(S_{E}-K_{E}\right)=0
$$

In order to make the calculation easy and convenient, the authors introduced non-dimensional variables as [13]

$$
X=\frac{x}{a}, \quad Y=\frac{y}{a}, \quad \bar{W}=\frac{w}{a}, \quad \bar{h}=\frac{h}{a},
$$

The expressions for the kinetic energy $\left(K_{E}\right)$ and the strain energy $\left(S_{E}\right)$ of an isotropic rectangular plate are as follows [8]:

$$
\begin{aligned}
& K_{E}=\frac{1}{2} p^{2} \int_{0}^{a} \int_{0}^{b} \rho h W^{2} d y d x, \\
& S_{E}=\frac{1}{2} \int_{0}^{a} \int_{0}^{b} D_{1}\left\{\left(W_{x x}\right)^{2}+\left(W_{y y}\right)^{2}+2 v\left(W_{x x}\right)\left(W_{y y}\right)+2(1-v)\left(W_{x y}\right)^{2}\right\} d y d x .
\end{aligned}
$$

Here, the suffix indicates partial differentiation.

On using the above assumptions along with Eq.(3.2); Eq.(3.3) and Eq.(3.4) become

$$
\begin{aligned}
& K_{E}=\frac{1}{2} \rho_{0} p^{2} \overline{h_{0}} a^{5} \int_{0}^{1} \int_{0}^{b / a}\left[\left(1+\alpha_{1} \sin (\pi X)\right)(1+\beta \sin (\pi X)) \bar{W}^{2}\right] d Y d X, \\
& S_{E}=\frac{E_{0} \bar{h}_{0}^{3} a^{3}}{24\left(1-v^{2}\right)} \int_{0}^{1} \int_{0}^{b / a}\left\{1-\alpha\left(1-\sin \left(\pi \frac{X}{2}\right)\right)\right\}(1+\beta \sin (\pi X))^{3} \times
\end{aligned}
$$

and

$$
\times\left[\left(\frac{\partial^{2} \bar{W}}{\partial X^{2}}\right)^{2}+\left(\frac{\partial^{2} \bar{W}}{\partial Y^{2}}\right)^{2}+2 v \frac{\partial^{2} \bar{W}}{\partial X^{2}} \frac{\partial^{2} \bar{W}}{\partial Y^{2}}+2(1-v)\left(\frac{\partial^{2} \bar{W}}{\partial X \partial Y}\right)^{2}\right] d Y d X
$$

Using Eq.(3.5) and Eq.(3.6) in Eq.(3.1), one gets [9]:

$$
\delta\left(S_{E}^{\#}-\lambda^{2} K_{E}^{\#}\right)=0
$$

where

$$
K_{E}^{\#}=\int_{0}^{1} \int_{0}^{b / a}\left[\left(1+\alpha_{1} \sin (\pi X)\right)(1+\beta \sin (\pi X)) \bar{W}^{2}\right] d Y d X,
$$


and

$$
S_{E}^{\#}=\int_{0}^{1} \int_{0}^{b / a}\left\{1-\alpha\left(1-\sin \left(\pi \frac{X}{2}\right)\right)\right\}(1+\beta \sin (\pi X))^{3} \times
$$

$$
\times\left[\left(\frac{\partial^{2} \bar{W}}{\partial X^{2}}\right)^{2}+\left(\frac{\partial^{2} \bar{W}}{\partial Y^{2}}\right)^{2}+2 v \frac{\partial^{2} \bar{W}}{\partial X^{2}} \frac{\partial^{2} \bar{W}}{\partial Y^{2}}+2(1-v)\left(\frac{\partial^{2} \bar{W}}{\partial X \partial Y}\right)^{2}\right] d Y d X .
$$

Here, $\lambda^{2}=\frac{12 \rho_{0} p^{2} a^{2}\left(1-v^{2}\right)}{E_{0} \bar{h}_{0}^{2}}$ is the expression of the required frequency parameter.

Equation (3.7) consists of two unknown constants, i.e. $A_{1}$ and $A_{2}$ arising due to the substitution of $W$. These two constants are to be determined as follows [17]

$$
\frac{\partial}{\partial A_{n}}\left(S_{E}^{*}-\lambda^{2} K_{E}^{*}\right)=0, \quad n=1,2 .
$$

From Eq.(3.10), the authors obtain the following

$$
b_{n 1} A_{1}+b_{n 2} A_{2}=0, \quad n=1,2
$$

where $b_{n 1}$ and $b_{n 2}$ include structural parameters.

For convenience, the authors took $A_{1}=1$. Now, the value of $A_{2}$ can be obtained easily. In order to get a non-trivial solution of Eq.(3.11), the determinant of Eq.(3.11) must be zero [18]

$$
\left|\begin{array}{ll}
b_{11} & b_{12} \\
b_{21} & b_{22}
\end{array}\right|=0
$$

Equation (3.12) is a bi-quadratic equation in $\lambda$. Various values of the frequency parameter for different values of the structural parameters are computed.

\section{Results and discussion}

Results are obtained for an alloy of aluminum, i.e. "Duralumin" which is a visco-elastic material. For Duralumin, the following parameters are used in the calculations:

$$
\begin{aligned}
& \mathrm{E}=7.08 \times 10^{10} \mathrm{~N} / \mathrm{M}^{2}, \quad G=2.632 \times 10^{10} \mathrm{~N} / \mathrm{M}^{2}, \quad \eta=14.612 \times 10^{5} \mathrm{Ns} / \mathrm{M}^{2}, \\
& \rho_{0}=2.8 \times 10^{3} \mathrm{~kg} / \mathrm{M}^{3}, \quad v=0.345 \quad \text { and } \quad h_{0}=0.01 \mathrm{M} .
\end{aligned}
$$

The first two modes of the frequency parameter are calculated for various combinations of the structural parameters at different boundary conditions. All numerical values of the frequency parameter are shown in Tabs 1-4.

In Tab.1, the first two modes of the frequency parameter at different values of the taper parameter are placed systematically for three boundary conditions. The authors discussed the following cases
(i)
$\alpha=0.0, \alpha_{1}=0.0, a / b=1.5$
(ii) $\alpha=0.2, \alpha_{1}=0.0, a / b=1.5$
(iii) $\alpha=0.6, \alpha_{1}=0.0, a / b=1.5$.

From Tab.1, the authors concluded the following 
i) For each fixed value of the taper parameter, the frequency parameter (for the first two modes of the vibration) decreases as the thermal gradient increases (at $\alpha_{1}=0.0, a / b=1.5$ ) from 0.0 to 0.6 , i.e. case (i) to case (iii) for all boundary conditions.

ii) For increasing values of the taper parameter (from 0.0 to 1.0 ), the frequency parameter (for all boundary conditions) increases continuously.

iii) The first mode of the frequency parameter is maximum for C-SS-C-SS and minimum for C-C-C-C at each value of the taper parameter for all the cases.

iv) The second mode of the frequency parameter is maximum for C-C-C-C and minimum for SS-SS-SS-SS at each value of the taper parameter for all the cases.

Table 1. Frequency v/s taper parameter.

\begin{tabular}{|c|c|c|c|c|c|c|c|}
\hline \multirow{2}{*}{ B.C.'s } & \multirow{2}{*}{$\beta$} & \multicolumn{2}{|c|}{$\alpha=0.0, \alpha_{1}=0.0, a / b=1.5$} & \multicolumn{2}{|c|}{$\alpha=0.2, \alpha_{1}=0.0, a / b=1.5$} & \multicolumn{2}{|c|}{$\alpha=0.6, \alpha_{1}=0.0, a / b=1.5$} \\
\hline & & Mode 1 & Mode 2 & Mode 1 & Mode 2 & Mode 1 & Mode 2 \\
\hline C-C-C-C & \multirow{3}{*}{0.0} & 256.20 & 64.82 & 247.70 & 62.65 & 229.78 & 58.07 \\
\hline SS-SS-SS-SS & & 256.91 & 34.25 & 248.05 & 33.05 & 229.31 & 30.50 \\
\hline C-SS-C-SS & & 288.81 & 41.94 & 279.51 & 40.46 & 259.90 & 37.33 \\
\hline C-C-C-C & \multirow{3}{*}{0.2} & 300.421 & 74.98 & 290.64 & 72.51 & 270.02 & 67.31 \\
\hline SS-SS-SS-SS & & 298.43 & 38.94 & 288.39 & 37.60 & 267.20 & 34.76 \\
\hline C-SS-C-SS & & 340.72 & 47.54 & 329.87 & 45.91 & 307.01 & 42.47 \\
\hline C-C-C-C & \multirow{3}{*}{0.4} & 345.79 & 85.41 & 334.67 & 82.63 & 311.22 & 76.78 \\
\hline SS-SS-SS-SS & & 341.70 & 43.81 & 330.41 & 42.32 & 306.58 & 39.18 \\
\hline C-SS-C-SS & & 393.41 & 53.45 & 380.97 & 51.65 & 354.76 & 47.86 \\
\hline C-C-C-C & \multirow{3}{*}{0.6} & 391.89 & 96.01 & 379.38 & 92.92 & 353.03 & 86.39 \\
\hline SS-SS-SS-SS & & 386.11 & 48.81 & 373.50 & 47.17 & 346.91 & 43.72 \\
\hline C-SS-C-SS & & 446.58 & 59.55 & 432.52 & 57.58 & 402.92 & 53.42 \\
\hline C-C-C-C & \multirow{3}{*}{0.8} & 438.47 & 106.73 & 424.54 & 103.31 & 395.23 & 96.10 \\
\hline SS-SS-SS-SS & & 431.28 & 53.90 & 417.31 & 52.10 & 387.87 & 48.32 \\
\hline C-SS-C-SS & & 500.06 & 65.79 & 484.36 & 63.63 & 451.33 & 59.08 \\
\hline C-C-C-C & \multirow{3}{*}{1.0} & 485.37 & 117.53 & 470.01 & 113.78 & 437.70 & 105.88 \\
\hline SS-SS-SS-SS & & 476.99 & 59.04 & 461.64 & 57.09 & 429.29 & 52.98 \\
\hline C-SS-C-SS & & 553.75 & 72.12 & 536.41 & 69.77 & 499.93 & 64.82 \\
\hline
\end{tabular}

In Tab.2, the frequency parameter (for the first two modes of the vibration) is calculated for increasing values of the thermal gradient from 0.0 to 0.8 at various boundary conditions for the following cases 
iv)

$\alpha_{1}=0.0, \beta=0.0, a / b=1.5$

v) $\alpha_{1}=0.0, \beta=0.2, a / b=1.5$

vi) $\alpha_{1}=0.0, \beta=0.6, a / b=1.5$.

The authors summarized the findings of Tab.2 as follows:

For each fixed value of the thermal gradient, the frequency parameter (for the first two modes of the vibration) increases continuously as values of the taper parameter increase from 0.0 to 0.6 , i.e. case (iv) to case (vi) for all boundary conditions.

i) For increasing values of the thermal gradient from 0.0 to 0.8 , the frequency parameter (for the first two modes of the vibration) decreases for all cases and boundary conditions.

ii) The first mode of the frequency parameter is maximum for C-SS-C-SS and minimum for C-C-C-C for all paired values of the thermal gradient and cases (iv) - (vi).

iii) The second mode of the frequency parameter is maximum for C-C-C-C and minimum for SS-SS-SS-SS for all paired values of the thermal gradient and cases (iv) - (vi).

Table 2. Frequency v/s thermal gradient.

\begin{tabular}{|c|c|c|c|c|c|c|c|}
\hline \multirow{2}{*}{ B.C.'s } & \multirow{2}{*}{$\alpha$} & \multicolumn{2}{|c|}{$\alpha_{1}=0.0, \alpha=0.0, a / b=1.5$} & \multicolumn{2}{|c|}{$\alpha_{1}=0.0, \alpha=0.2, a / b=1.5$} & \multicolumn{2}{|c|}{$\alpha_{1}=0.0, \beta=0.6, a / b=1.5$} \\
\hline & & Mode 1 & Mode 2 & Mode 1 & Mode 2 & Mode 1 & Mode 2 \\
\hline C-C-C-C & \multirow{3}{*}{0.0} & 256.20 & 64.82 & 300.42 & 74.98 & 391.89 & 96.01 \\
\hline SS-SS-SS-SS & & 256.91 & 34.25 & 298.43 & 38.94 & 386.11 & 48.81 \\
\hline C-SS-C-SS & & 288.81 & 41.94 & 340.72 & 47.54 & 446.58 & 59.55 \\
\hline C-C-C-C & \multirow{3}{*}{0.2} & 257.70 & 62.65 & 290.64 & 72.51 & 379.38 & 92.92 \\
\hline SS-SS-SS-SS & & 248.05 & 33.05 & 288.39 & 37.60 & 373.50 & 47.17 \\
\hline C-SS-C-SS & & 279.51 & 40.46 & 329.87 & 45.91 & 432.52 & 57.58 \\
\hline C-C-C-C & \multirow{3}{*}{0.4} & 238.91 & 60.41 & 280.52 & 69.96 & 366.44 & 89.71 \\
\hline SS-SS-SS-SS & & 238.86 & 31.80 & 278.00 & 36.20 & 360.45 & 45.48 \\
\hline C-SS-C-SS & & 269.88 & 38.93 & 318.64 & 44.22 & 417.98 & 55.54 \\
\hline C-C-C-C & \multirow{3}{*}{0.6} & 229.78 & 58.05 & 270.02 & 67.31 & 353.03 & 86.39 \\
\hline SS-SS-SS-SS & & 229.31 & 30.50 & 267.02 & 34.76 & 346.91 & 43.72 \\
\hline C-SS-C-SS & & 259.90 & 37.33 & 307.01 & 42.47 & 402.92 & 53.42 \\
\hline C-C-C-C & \multirow{3}{*}{0.8} & 220.27 & 55.64 & 259.10 & 64.55 & 339.08 & 82.93 \\
\hline SS-SS-SS-SS & & 219.33 & 29.13 & 255.95 & 33.25 & 332.81 & 41.88 \\
\hline C-SS-C-SS & & 249.52 & 35.67 & 294.91 & 40.64 & 387.27 & 51.21 \\
\hline
\end{tabular}

To observe the effect of the non-homogeneity constant on the frequency parameter, the authors computed the frequency parameter with increasing values of the non-homogeneity constant $\left(\alpha_{1}\right)$ for the following cases:

(vii)

$$
\alpha=0.0, \beta=0.0, a / b=1.5 \quad \text { (viii) } \alpha=0.4, \beta=0.4, a / b=1.5
$$

(ix) $\alpha=0.8, \beta=0.8, a / b=1.5$.

Results for case (vii), case (viii) and case (ix) are presented in Tab.3. The authors analyzed the numerical results in Tab.3 in the following manner: 
i) For all the values of $\alpha_{1}$ the frequency parameter for both the modes of the vibration is minimum for case (vii) and maximum for case (ix) at corresponding boundary conditions.

ii) The first mode of the frequency parameter is maximum for C-SS-C-SS and minimum for C-C-C-C while the second mode of the frequency parameter is maximum for C-C-C-C and minimum for SS-SSSS-SS for all the corresponding values of the structural parameters.

iii) It is interesting to note that both the modes of the frequency parameter increase as the non-homogeneity constant varies from 0.0 to 1.0 for all the cases. It also proves that the non-homogeneity in the plate material directly affects the vibration of the rectangular plate.

Table 3. Frequency v/s non-homogeneity constant.

\begin{tabular}{|c|c|c|c|c|c|c|c|}
\hline \multirow{2}{*}{ B.C.'s } & \multirow{2}{*}{$\alpha_{1}$} & \multicolumn{2}{|c|}{$\alpha=0.0, \beta=0.0, a / b=1.5$} & \multicolumn{2}{|c|}{$\alpha=0.4, \beta=0.4, a / b=1.5$} & \multicolumn{2}{|c|}{$\alpha=0.8, \beta=0.8, a / b=1.5$} \\
\hline & & Mode 1 & Mode 2 & Mode 1 & Mode 2 & Mode 1 & Mode 2 \\
\hline C-C-C-C & \multirow{3}{*}{0.0} & 256.20 & 64.82 & 323.16 & 79.76 & 379.72 & 92.28 \\
\hline SS-SS-SS-SS & & 256.91 & 34.25 & 318.72 & 40.78 & 372.28 & 46.31 \\
\hline C-SS-C-SS & & 288.81 & 41.94 & 368.10 & 49.79 & 433.87 & 56.67 \\
\hline $\mathrm{C}-\mathrm{C}-\mathrm{C}-\mathrm{C}$ & \multirow{3}{*}{0.2} & 279.17 & 71.53 & 352.65 & 88.04 & 414.72 & 101.89 \\
\hline SS-SS-SS-SS & & 277.71 & 37.59 & 345.19 & 44.79 & 403.72 & 50.90 \\
\hline C-SS-C-SS & & 316.59 & 46.30 & 403.95 & 55.00 & 476.45 & 62.61 \\
\hline C-C-C-C & \multirow{3}{*}{0.4} & 309.99 & 80.71 & 392.38 & 99.58 & 462.01 & 115.29 \\
\hline SS-SS-SS-SS & & 305.01 & 42.14 & 380.11 & 50.28 & 445.32 & 57.18 \\
\hline C-SS-C-SS & & 354.50 & 52.39 & 453.06 & 62.28 & 534.90 & 70.93 \\
\hline $\mathrm{C}-\mathrm{C}-\mathrm{C}-\mathrm{C}$ & \multirow{3}{*}{0.6} & 354.93 & 95.15 & 450.65 & 117.29 & 531.60 & 135.89 \\
\hline SS-SS-SS-SS & & 343.73 & 48.86 & 429.91 & 58.44 & 504.88 & 66.56 \\
\hline C-SS-C-SS & & 411.02 & 61.76 & 526.66 & 73.51 & 622.77 & 83.79 \\
\hline C-C-C-C & \multirow{3}{*}{0.8} & 432.60 & 121.17 & 552.22 & 149.70 & 653.47 & 173.74 \\
\hline SS-SS-SS-SS & & 408.14 & 60.35 & 513.23 & 72.52 & 604.94 & 82.86 \\
\hline C-SS-C-SS & & 511.09 & 79.02 & 658.03 & 94.34 & 780.41 & 107.74 \\
\hline C-C-C-C & \multirow{3}{*}{1.0} & 708.89 & 194.13 & 919.35 & 242.51 & 1097.70 & 293.77 \\
\hline SS-SS-SS-SS & & 599.31 & 87.33 & 764.19 & 106.64 & 908.80 & 123.15 \\
\hline C-SS-C-SS & & 843.85 & 130.81 & 1102.79 & 158.44 & 1319.90 & 182.63 \\
\hline
\end{tabular}

In order to analyze the effect of the size of the rectangular plate on the vibrations of the plate, the authors evaluated the frequency parameter (for the first two modes of vibration) at different values of the aspect ratio of the plate (ratio of the length to the width of the rectangular plate) at various combinations of the structural parameters for different boundary conditions and tabulated all numerical values in Tab.4. Here, the authors considered the following three cases: 
$(\mathrm{x})$

$$
\alpha=\alpha_{I}=\beta=0.0 \quad \text { (xi) } \quad \alpha=\alpha_{I}=\beta=0.4 \quad \text { (xii) } \quad \alpha=\alpha_{I}=\beta=0.8 \text {. }
$$

On the basis of Tab.4, the following pragmatic observations were made:

i) As the aspect ratio increases from 0.5 to 1.5 , the frequency parameter (for the first two modes of vibration) increases continuously for all cases.

ii) For each value of the aspect ratio, the frequency parameter (for the first two modes of vibration) increases as the structural parameters vary from case (x) to case (xii) at corresponding boundary conditions.

iii) It is clearly seen that the frequency parameter in case (x) is minimum and it increases from case (x) to case (xii).

Table 4. Frequency v/s aspect ratio.

\begin{tabular}{|c|c|c|c|c|c|c|c|}
\hline \multirow{2}{*}{ B.C.'s } & \multirow{2}{*}{$a / b$} & \multicolumn{2}{|c|}{$\alpha=\alpha_{1}=\beta=0.0$} & \multicolumn{2}{|c|}{$\alpha=\alpha_{I}=\beta=0.4$} & \multicolumn{2}{|c|}{$\alpha=\alpha_{1}=\beta=0.8$} \\
\hline & & Mode 1 & Mode 2 & Mode 1 & Mode 2 & Mode 1 & Mode 2 \\
\hline C-C-C-C & \multirow{3}{*}{0.5} & 104.94 & 26.24 & 137.57 & 34.50 & 213.84 & 53.16 \\
\hline SS-SS-SS-SS & & 105.91 & 13.17 & 123.43 & 19.39 & 169.11 & 31.30 \\
\hline C-SS-C-SS & & 86.90 & 25.38 & 117.57 & 33.56 & 190.75 & 52.78 \\
\hline C-C-C-C & \multirow{3}{*}{1} & 150.10 & 38.35 & 217.12 & 55.63 & 354.06 & 93.67 \\
\hline SS-SS-SS-SS & & 149.67 & 21.08 & 202.85 & 30.54 & 309.52 & 49.71 \\
\hline C-SS-C-SS & & 151.36 & 30.95 & 228.62 & 43.80 & 388.52 & 73.68 \\
\hline C-C-C-C & \multirow{3}{*}{1.5} & 256.20 & 64.82 & 392.38 & 99.58 & 653.47 & 173.74 \\
\hline SS-SS-SS-SS & & 256.91 & 34.25 & 380.11 & 50.28 & 604.94 & 82.86 \\
\hline C-SS-C-SS & & 288.81 & 41.94 & 453.06 & 62.28 & 780.41 & 107.74 \\
\hline
\end{tabular}

\section{Conclusions}

On the basis of the above results and discussion, the authors conclude that an acute change in the structural parameter may affect the vibrational properties of the rectangular plate. The main goal of this study is to provide basic information about vibrations of the rectangular plate at various combinations of the structural parameters. The authors concluded the findings of this paper as follows:

i) The frequency parameter (for the first two modes of vibration) is maximum for zero effect of temperature, i.e. $\alpha=0.0$ for all the values of the taper parameter.

ii) The frequency parameter is minimum for uniform thickness of the rectangular plate, i.e. $\beta=0.0$ while it increases as the taper parameter increases from zero to a non-zero value.

iii) The frequency parameter is minimum for the homogeneous rectangular plate, i.e. $\alpha_{1}=0.0$ while it increases as the non-homogeneity constant increases from zero to a non-zero value.

iv) The frequency parameter is maximum at $a / b=1.5$ and minimum at $a / b=0.5$. The size of the plate directly affects the vibrations of the rectangular plate.

\section{Nomenclature}

$a-$ length of rectangular plate
$b-$ breadth of rectangular plate 


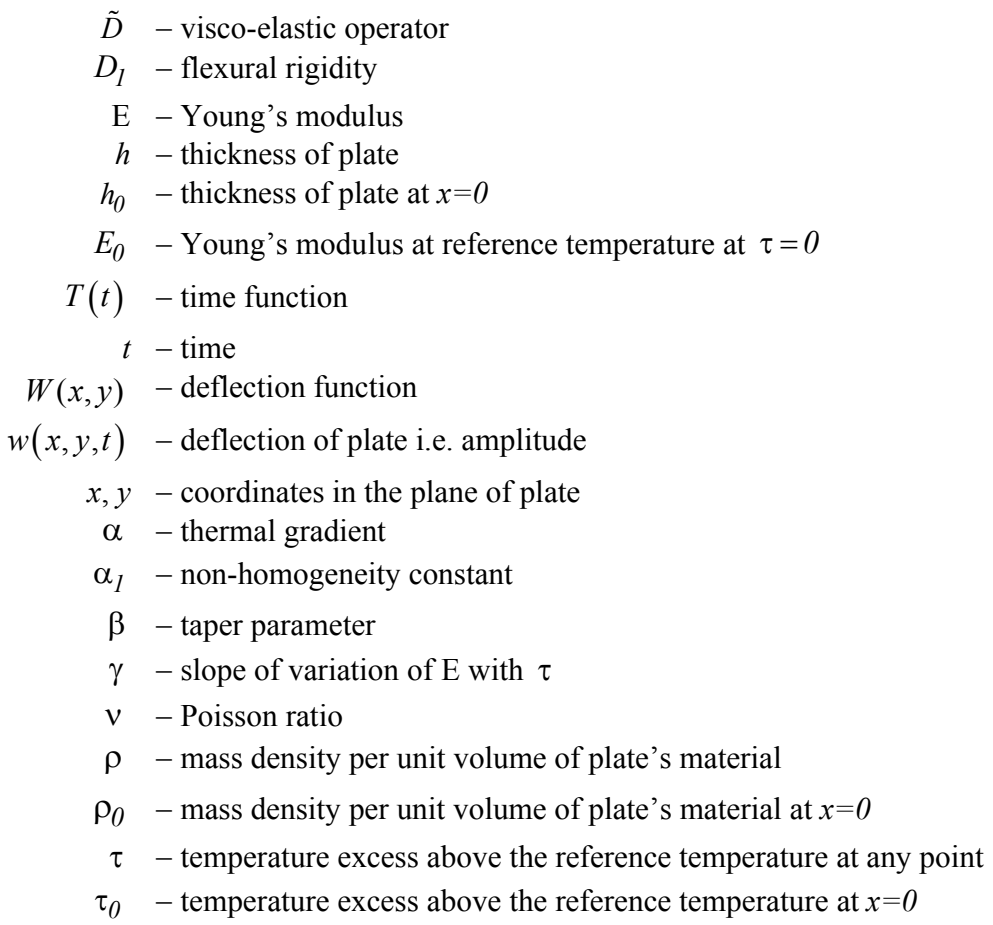

\section{References}

[1] Leissa A.W. (1969): Vibration of Plates. - Washington, D.C.: NASA.

[2] Sobotka Z. (1978): Free vibration of visco-elastic orthotropic rectangular plates. - Acta Technica (CSAV), vol.6, pp.678705.

[3] Tomar J.S. and Gupta A.K. (1983): Thermal effect on frequencies of an orthotropic rectangular plate of linearly varying thickness. - Journal of Sound and Vibration, vol.90, No.3, pp.325-331.

[4] Tomar J.S., Gupta D.C. and Jain N.C. (1984): Free vibrations of an isotropic non-homogeneous infinite plate of parabolically varying thickness. - Indian Journal of Pure and Applied Mathematics, vol.15, No.2, pp.211-220.

[5] Cheung Y.K. and Zhou D. (1999): The free vibrations of tapered rectangular plates using a new set of beam functions with the Rayliegh-Ritz method. - Journal of Sound and Vibration, vol.223, No.5, pp.703-722.

[6] Lal R. (2003): Transverse vibrations of orthotropic non-uniform rectangular plates with continuously varying density. - Indian Journal of Pure and Applied Mathematics, vol.34, No.4, pp.587-606.

[7] Li W.L. (2004): Vibration analysis of the rectangular plate with general elastic boundary supports. - Journal of Sound and Vibration, vol.273, No.3, pp.619-635.

[8] Gupta A.K., Kumar M., Kumar S. and Khanna A. (2011): Thermal effect on vibration of parallelogram plate of bidirection linearly varying thickness. - Applied Mathematics, vol.1, No.2, pp.33-38.

[9] Gupta A.K. and Sharma P. (2013): Thermal analysis on frequencies of non-homogeneous trapezoidal plate of variable thickness and density. - Acta Tecnica, vol.58, No.2, pp.189-205.

[10] Khanna A. and Singhal A. (2015): A study on free vibration of visco-elastic tapered plate with clamped ends. Romanian Journal of Acoustics and Vibration, vol.12, No.1, pp.43-48.

[11] Chakraverty S. (2009): Vibration of Plates. - Florida: Taylor and Francis.

[12] Gupta A.K., Agarwal N. and Kaur H. (2011): Transverse vibration of non-homogeneous orthotropic visco-elastic circular plate of varying parabolic thickness. - Mathematical Methods in the Applied Sciences, vol.34, pp.20652076. 
[13] Khanna A. and Singhal A. (2013): An analytical approach on thermally induced vibrations of non-homogeneous tapered plate. - Journal of Mathematics, vol. 2013, Article ID 721868, 6 pages.

[14] Khanna A. and Kaur N. (2016): Effect of thermal gradient on vibration of non-uniform visco-elastic rectangular plate. - Journal of the Institution of Engineers (India): Series C, vol.97, No.2, pp.141-148.

[15] Khanna A. and Kaur N. (2016): Effect of structural parameters on vibration of non-homogeneous visco-elastic rectangular plate. - Journal of Vibration Engineering and Technologies, vol.4, No.5, pp.459-466.

[16] Khanna A. and Singhal A. (2016): Effect of bi-directional temperature variation on vibration of rectangular plate with bi-parabolic thickness variation. - Journal of Low Frequency Noise, Vibration and Active Control, vol.35, No.2, pp.139-151.

[17] Singhal A. (2016): Vibrational Behavior of Visco-Elastic Tapered Rectangular Plate. - Ph.D. Thesis, M.M.D.U.Mullana, India.

[18] Khanna A. and Kaur N. (2014): A study on vibration of tapered rectangular plate under non-uniform temperature field. - Mechanika, vol.20, No.4, pp.376-381.

Received: December 27, 2017

Revised: June 15, 2018 